\title{
Análisis de la influencia de las señales del sistema GLONASS en el procesamiento de una red geodésica regional
}

\author{
Analysis of the influence of GLONASS signals in the processing of one regional \\ geodestic Network
}

\section{Análise da influência dos sinais do sistema GLONASS no processamento de uma rede geodésica regional}

\author{
Diana Paniagua-Jiménez \\ diananinette21@gmail.com \\ Escuela de Topografía, Catastro y Geodesia \\ Universidad Nacional \\ Heredia, Costa Rica \\ Jose Valverde-Calderón \\ jose.valverde.calderon@una.cr \\ Escuela de Topografía, Catastro y Geodesia \\ Universidad Nacional \\ Heredia, Costa Rica
}

Recibido-Received: 2/may/ 2016 / Corregido-Corrected: 18/mar/2017.

Aceptado-Accepted: 21/abr/ 2017 / Publicado-Published: 31/ene/2018.

\begin{abstract}
Resumen
En los últimos años, ha habido un incremento en el número de satélites disponibles para posicionamiento mediante técnicas espaciales, donde se destacan los sistemas GPS y GLONASS. Se realizó el análisis comparativo de los resultados obtenidos tras el cálculo de una red de estaciones GNSS en Europa, a partir del procesamiento de los datos usando el programa Bernese 5.2 que, entre otras cosas, destaca por presentar una mejora en el tratamiento conjunto de datos GPS y GLONNAS, con respecto a la versión anterior del programa. El fin primario que motivó la investigación es la necesidad de determinar si efectivamente los resultados de la combinación de datos de ambos sistemas son mejores que los obtenidos con la versión 5.0, ya que investigaciones demostraron que el resultado del procesamiento GNSS en el programa Bernese 5.0 tiene la tendencia a ser menos preciso que el resultado del procesamiento con únicamente datos GPS. Como resultado se obtuvo que el incluir las observables del sistema GLONASS en el procesamiento no tiene un impacto significativo sobre los resultados.
\end{abstract}

Palabras claves: Geodesia; Bernese; GPS; GLONASS. 
UNICIENCIA Vol. 32, No. 1, pp. 1-17. Enero-Junio, 2018.

ISSN Electrónico: 2215-3470

URL: www.revistas.una.ac.cr/uniciencia

DOI: http://dx.doi.org/10.15359/ru.32-1.1

Email: revistauniciencia@una.cr

\begin{abstract}
In recent years, there has been an increase in the number of satellites available for positioning using space techniques, highlighting the GPS and GLONASS systems. The comparative analysis of the results obtained from the calculation of a network of GNSS stations in Europe, from data processing using the Bernese 5.2 software, which, among other things, stand out for presenting an improvement in the joint treatment of GPS and GLONASS data with respect to the previous version of the program. The primary purpose that motivated the research is the need to determine whether or not the results of the combination of data from both systems are better than those obtained with version 5.0, since research has shown that the GNSS processing result in the Bernese 5.0 program tends to be less accurate than the result of processing with only GPS data. Consequently, it was found that the inclusion of GLONASS observables in the processing does not impact significantly on the results.
\end{abstract}

Keywords: Geodesy; Bernese; GPS; GLONASS.

\begin{abstract}
Resumo
Nos últimos anos tem aumentado o número de satélites disponíveis para posicionamento através de técnicas espaciais, onde se destacam os sistemas GPS e GLONASS. Realizou-se a análise comparativa dos resultados obtidos depois do cálculo de uma rede de estações GNSS na Europa, a partir do processamento dos dados usando o programa Bernese 5.2 que, entre outras coisas, destaca-se por apresentar uma melhora no tratamento conjunto de dados GPS e GLONNAS, com respeito à versão anterior do programa. A finalidade primária que motivou a pesquisa é a necessidade de determinar se efetivamente os resultados da combinação de dados de ambos os sistemas são melhores que os obtidos com a versão 5.0, já que pesquisas demostraram que o resultado do processamento GNSS no programa Bernese 5.0 tem a tendência de ser menos preciso do que o resultado do processamento com unicamente dados GPS. Como resultado obteve-se que incluir as observáveis do sistema GLONASS no processamento não tem um impacto significativo sobre os resultados.
\end{abstract}

Palavras-chaves: Geodesia; Bernese; GPS; GLONASS.

Con la creciente disponibilidad de receptores GNSS, la revitalización de GLONASS con el aumento de satélites en su constelación y la publicación de órbitas por el IGS para GLONASS con una latencia de varias semanas, se ha convertido en una tarea importante estudiar el uso de los datos GLONASS para las rutinas de análisis de datos de la EPN y en general, para las redes continentales (Bruyninx, 2006).

En 2006 en el Observatorio Real de Bélgica se realizó una investigación con el fin de estudiar las ventajas y desventajas del análisis combinado con datos de los sistemas GPS y GLONASS para una red regional usando Bernese v5.0. Luego del procesamiento, los resultados mostraron diferencias entre las coordenadas con datos solamente GPS y las coordenadas con datos GNSS en un nivel de 1-2 $\mathrm{mm}$ en la componente horizontal y entre $2 \mathrm{~mm}$ y $6 \mathrm{~mm}$ en vertical. La causa de las discrepancias no fue clara y se recomendó efectuar más estudios (Bruyninx, 2006).

En la actualidad, la comunidad científica global y en general las geociencias aceptan el ITRF como marco de referencia según la resolución 2 dada por la Unión Internacional de Geodesia y Geofísica (UIGG, por sus siglas en inglés) tras su asamblea general en 2007 (Petit \& Luzum, 2010). Esto implica que el ITRF debe ser mantenido en el tiempo, a través de la medición continua en las estaciones escogidas, así como de su procesamiento y seguimiento continuo.

Para definir el ITRF, se recurre a la combinación de las observaciones de varias técnicas de la geodesia espacial, donde se destaca el Sistema de Posicionamiento Global (GPS, por sus 
siglas en inglés), el cual, en primera instancia, es un sistema para la navegación (HofmmannWellenhof, Lichtenegger \& Wasle, 2008).

Considerando la puesta en operación de otros sistemas de navegación global, como el administrado por la Federación Rusa denominado GLONASS, que tiene actualmente 24 satélites operativos o el sistema Galileo, de la Agencia Espacial Europea (ESA, por sus siglas en inglés), el cual cuando esté completamente operativo dispondrá de 30 satélites adicionales, se hace necesario investigar cuál es la mejor forma de combinar las observaciones del "aceptado" GPS con las observaciones de otros sistemas satelitales de posicionamiento, como GLONASS y GALILEO.

La Subcomisión 1.3a de la IAG, a cargo del Marco de Referencia para Europa (EUREF, por sus siglas en inglés) también ha concluido que al procesar estaciones solo GPS y luego usar datos combinados GNSS, usando el programa Bernese V5.0, las coordenadas pueden variar en los componentes horizontales entre 1 y $2 \mathrm{~mm}$ y en la vertical hasta $6 \mathrm{~mm}$ con respecto a la solución GPS (Habrich, 2009).

Considerando que desde agosto de 2013 se publicó la versión 5.2 del programa Bernese, en cuyas características, de acuerdo con Dach \& Walser (2013), indica una mejora en el tratamiento conjunto de datos GNSS, así como la necesidad de generar experiencias en este tipo de procesamiento, se realizó un proyecto de investigación cuyo principal objetivo fue efectuar el análisis comparativo de los resultados obtenidos tras el cálculo de una red de estaciones GNSS en Europa, a partir del procesamiento de únicamente datos GPS y GPS+GLONASS, usando el programa Bernese 5.2. Esto permitiría, entre otras cosas, determinar los posibles beneficios de incluir observaciones GLONASS en investigaciones relacionadas con el análisis de la deformación superficial tras terremotos, estudios de la troposfera, entre otras aplicaciones.

\section{Marco teórico}

\section{Definición de geodesia}

La geodesia es la ciencia que se encarga de estudiar la forma y dimensiones de la Tierra, así como su campo de gravedad (Torge, 2001). Los problemas básicos que debe resolver, de acuerdo con Seeber (2003) son:

1. La determinación de posiciones tridimensionales precisas a una escala global, regional y local (establecimiento de control geodésico para aplicaciones científicas e ingeniería)

2. Determinación del campo de gravedad de la Tierra y las funciones lineales de este campo (un geoide preciso y modelos de geopotencial)

3. Medición y modelado de fenómenos geodinámicos (variaciones del movimiento del polo y rotación de la Tierra, deformaciones de la corteza, estudios atmosféricos, entre otros).

\section{Sistemas y marcos geodésicos de referencia}

La geodesia requiere de marcos de referencia consistentes, exactos y confiables, para la medición y mapeo de la superficie de la Tierra y sus variaciones en el tiempo. Los marcos geodésicos de referencia son la base para gran cantidad de aplicaciones prácticas tales como redes nacionales que sustentan la cartografía de un país, la navegación precisa, el mantenimiento de sistemas de información geoespacial, así también como para aplicaciones científicas relacionadas con el estudio de fenómenos de naturaleza geofísica que ocurren en la Tierra. Las técnicas de 
UNICIENCIA Vol. 32, No. 1, pp. 1-17. Enero-Junio, 2018.

observación de la geodesia espacial permiten la determinación de parámetros geodésicos con una exactitud de varios milímetros (Angermann et al., 2003).

Un sistema de referencia es la definición conceptual completa de cómo un sistema coordenado está formado. Define el origen y orientación de los planos fundamentales o ejes de este. También incluye los modelos físicos y matemáticos fundamentales. Se denominan convencionales cuando los modelos, constantes numéricas y algoritmos son especificados (Seeber, 2003).

Un marco de referencia es la realización práctica del sistema de referencia a través de observaciones. El marco de referencia terrestre (TRF, por sus siglas en inglés) es elaborado usando observaciones de la geodesia espacial y es accesible para los sujetos usuarios a través de valores numéricos, los cuales consisten en posiciones como una función del tiempo de una red de estaciones geodésicas. El TRF es la realización del sistema de referencia terrestre (TRS, por sus siglas en inglés) (Altamini, Collilieux \& Métivier, 2011).

\section{IGb08}

En noviembre de 2006, un marco de referencia basado en el ITRF2005, llamado IGS05, fue adoptado por el Servicio GNSS Internacional (IGS, por sus siglas en inglés); junto con el cambio del marco, se adoptó un modelo de calibración absoluto para los centros de fase de las antenas (Schmid, Steigenberger, Gendt, Ge \& Rothacher, 2007).

Debido a la propagación de errores y discontinuidades que afectaron la posición de muchas estaciones fiduciales, el IGS05 se volvió obsoleto. El 17 de abril de 2011, el IGS dejó de utilizar el IGS05 y estableció uno nuevo llamado IGS08, para sus productos. Este nuevo marco de referencia, basado en el ITRF2008, fue diseñado y oficialmente adoptado por el IGS desde la semana GPS 1632 (Rebischung et al., 2011).

Por cambios en la geometría de la red del IGS08, la calidad de esta decreció gradualmente en el tiempo. Esto resultó en la necesidad de actualizarla, de forma que los productos generados por el IGS no afectaran las soluciones del ITRF y las propias (Rebischung et al., 2011).

Debido a estaciones decomisadas, inactivas o que fueron afectadas por discontinuidades desde la época 2009,5, estas se convirtieron en inutilizables para ser usadas como fiduciales dentro de los productos del IGS. En particular, la red núcleo del IGS08 perdió alrededor de 50 estaciones, provocando vacíos en Suramérica, África y el este de Asia. Como solución a esta problemática se estableció el IGb08, el cual reemplazó oficialmente el IGS08 desde la semana GPS 1709. Este cambio ayudó a estabilizar el alineamiento del marco de referencia de los productos del IGS (P. Rebischung, comunicación personal, 24 de septiembre de 2012).

\section{Bernese v5.2}

La versión de Bernese 5.2 es nombrada como programa Bernese GNSS, ya que tiene la capacidad de resolver ambigüedades para GPS y GLONASS, además puede procesar datos del sistema Galileo, generar modelos ionosféricos entre otros (Dach et al., 2007).

La versión 5.2 fue liberada el 18 de diciembre del 2012 y fue elaborada por el Instituto de Astronomía de la Universidad de Berna (AIUB, por sus siglas en alemán); el software es el utilizado por el Centro para la Determinación de la Órbita de Europa (CODE, por sus siglas en inglés) en sus actividades para el IGS y las europeas (EUREF / EPN) (Walser, 2014). 
Bernese es un programa científico de procesamiento de datos GNSS, el cual incorpora sofisticadas herramientas las cuales toman en cuenta estándares de cálculo para aplicaciones geodésicas que estén basadas en el uso de observaciones GNSS (Dach et al., 2007).

En 2010, el IERS publicó un nuevo conjunto de convenciones para el cálculo y modelado de distintos fenómenos que deben ser tomados en cuenta para un procesamiento con fines geodésicos (Petit \& Luzum, 2010). Por tanto, ante esta situación fue necesario actualizar los modelos disponibles en la versión 5.0 de Bernese con el fin de hacerlas consistentes con las del IERS del año 2010 (Dach \& Walser, 2013).

La versión de Bernese 5.2 dispone de nuevas características, entre las que se destacan (Dach \& Walser, 2013):

1. Resolución de ambigüedades para GLONASS

2. Estimación de las correcciones del reloj a partir de datos GLONASS

3. Modelado de troposfera: GMF/ GPT, VMF1

4. Modelado de ionosfera: corrección de la ionosfera en orden superior

5. ADDNEQ2: cálculo de la repetibilidad con parámetros de transformación

6. ADDNEQ2: el SINEX contiene NEQ

7. Estimación del modelo de antena del receptor en ADDNEQ2

8. Aplicación de las normas del IGS y las convenciones del IERS de 2010

9. Análisis automatizado de las series de tiempo (FODITS)

10. ORBGEN: pulsos estocásticos para ajustar las órbitas

11. Introducir correcciones para la carga de la presión atmosférica

\section{EUREF}

EUREF es el componente europeo de la Comisión 1 de la IAG, a través de la Sub-Comisión 1.3 - Marcos de Referencia Regionales -. Sus actividades están relacionadas con la definición, realización y mantenimiento del marco de referencia en ese continente. Se enfoca en el componente geométrico y vertical (Agria et al., 2009).

De acuerdo con los autores citados en el párrafo anterior, EUREF se encarga de:

1. La realización del ETRS89 y la relación con el ITRF

2. La EPN, que contribuye con el mantenimiento del marco de referencia terrestre europeo (ETRF, por sus siglas en inglés), el ITRF y proyectos especiales relacionados

3. Actividades en tiempo real y el desarrollo de estándares y el medio operativo para distribuir datos GNSS vía internet

4. El Sistema de Referencia Vertical Europeo (EVRS, por sus siglas en inglés), incluyendo el estado de la Red de Nivelación Unificada Europea (UELN, por sus siglas en inglés) y la Acción de Densificación Vertical Unificada Europea (EUVN DA, por sus siglas en inglés)

5. El estado de la adopción del ETRS89 por los países y organizaciones europeas, como sistema de referencia oficial desde su definición en el año 1990. 
UNICIENCIA Vol. 32, No. 1, pp. 1-17. Enero-Junio, 2018.

ISSN Electrónico: 2215-3470

URL: www.revistas.una.ac.cr/uniciencia

DOI: http://dx.doi.org/10.15359/ru.32-1.1

Email: revistauniciencia@una.cr

\section{Metodología}

\section{Diseño de la red de estaciones de medición continua}

Para la red se seleccionó un área de trabajo con estaciones de medición continua donde los receptores registren observaciones de las constelaciones GPS y GLONASS. Otra condición fue que los datos fueran gratuitos para los usuarios y la distribución de estas, ya que se requieren líneas bases menores a $2000 \mathrm{~km}$ de longitud para que puedan ser resueltas usando la estrategia de procesamiento QIF en el programa Bernese V5.2 (Dach et al., 2007). La red procesada con solo datos GPS y que aplica la estrategia antes mencionada para la resolución de ambigüedades es la que se considera como de referencia.

En un análisis previo, se consideró que el área que cumple con dichos requisitos es Europa, específicamente la red que materializa el marco de referencia de Europa EUREF, por lo que se seleccionaron preliminarmente 27 estaciones de medición continua, ubicadas en distintos países: Bélgica, Alemania, Francia, Rumania, Grecia, Ucrania, Reino Unido, Italia, España, Letonia, República Checa, Austria, Estonia, Polonia, Suecia, Macedonia, Hungría, y Rusia.

La selección definitiva (ver figura 1) se hizo con base en el análisis del registro de mediciones de la estación, para lo cual se ingresó al sitio web de EUREF (www.epncb.oma.be) y se buscó información sobre ellos. Esto se encontró en un calendario que muestra la disponibilidad de observaciones. Luego del estudio, se descartaron sitios que no cumplían con los requisitos establecidos, los cuales registraran datos de los sistemas GPS y GLONASS para el periodo de procesamiento; como resultado de este paso, se definió la red final la cual es de 25 estaciones. En la tabla 1 se indica información referente a la red seleccionada para el proyecto.

Tabla 1

Metadatos de las estaciones que definen la red GNSS del proyecto

\begin{tabular}{lcll}
\hline Estación & Domes & \multicolumn{1}{c}{ Ubicación } & \multicolumn{1}{c}{ Institución } \\
\hline AJAC & 10077M005 & France-Ajaccio & Instituto Geográfico Nacional de Francia \\
BRST & $10004 \mathrm{M} 004$ & France-Brest & Instituto Geográfico Nacional de Francia \\
BUCU & $11401 \mathrm{M} 001$ & Romania-Bucuresti & Agencia Federal de Cartografía y Geodesia de Alemania \\
DYNG & $12602 \mathrm{M} 006$ & Greece-Dionysos & Centro Nacional de Estudios Espaciales de Francia \\
FFMJ & $14279 \mathrm{M} 001$ & Germany-Frankfurt & Agencia Federal de Cartografía y Geodesia de Alemania \\
GLSV & $12356 \mathrm{M} 001$ & Ukraine- Kiev & Academia Nacional de Ciencias de Ucrania \\
GOPE & $11502 \mathrm{M} 002$ & Czech Republic-Ondrejov & Observatorio Geodésico Pecny \\
GRAS & $10002 \mathrm{M} 006$ & France-Caussols & Centro Nacional de Estudios Espaciales de Francia \\
GRAZ & $11001 \mathrm{M} 002$ & Austria-Graz & Oficina Federal de Metrología y Topografía de Austria \\
HUEG & $14280 \mathrm{M} 001$ & Germany-Huegelheim & Agencia Federal de Cartografía y Geodesia de Alemania \\
KURE & 10604 S001 & Estonia-Kuressaare & Junta Directiva de Terrenos de Estonia \\
LAMA & $12209 \mathrm{M} 001$ & Poland-Olsztyn & Instituto de Geodesia de la Universidad de Warmia \\
LIL2 & $10051 \mathrm{M} 003$ & France-Lille & Instituto Geográfico Nacional de Francia \\
MARS & $10073 \mathrm{M} 008$ & France-Marseille & Instituto Geográfico Nacional de Francia
\end{tabular}




\begin{tabular}{lcll}
\hline Estación & Domes & \multicolumn{1}{c}{ Ubicación } & \multicolumn{1}{c}{ Institución } \\
\hline MAR6 & $10405 \mathrm{M} 002$ & Sweden-Maartsbo & Autoridad Sueca de Registración de Tierras y Catastro \\
MATE & $12734 \mathrm{M} 008$ & Italy-Matera & Agencia Espacial Italiana \\
ONSA & $10402 \mathrm{M} 004$ & Sweden-Onsala & Autoridad Sueca de Registración de Tierras y Catastro \\
ORID & $15601 \mathrm{M} 001$ & Macedonia-Ohrid & Agencia Federal de Cartografía y Geodesia de Alemania \\
PENC & $11206 \mathrm{M} 006$ & Hungary-Penc & Observatorio Geodésico Satelital FÖMI \\
POTS & $14106 \mathrm{M} 003$ & Germany-Potsdam & Centro Alemán de Investigación para Geociencias \\
SVTL & $12350 \mathrm{M} 001$ & Russian Federation-Svetloe & Instituto de Astronomía Aplicada RAS \\
SWKI & $12228 \mathrm{M} 001$ & Poland-Suwalki & Centro de Administración ASG-EUPOS Polonia \\
TLSE & $10003 \mathrm{M} 009$ & France-Toulouse & Centro Nacional de Estudios del Espacio \\
VILL & $13406 \mathrm{M} 001$ & Spain-Villafranca & Agencia Espacial Europea \\
WTZR & $14201 \mathrm{M} 010$ & Germany-Bad Koetzting & Agencia Federal de Cartografía y Geodesia de Alemania \\
\hline
\end{tabular}

Nota: Elaboración propia a partir de los log files disponibles en http://www.epncb.oma.be/

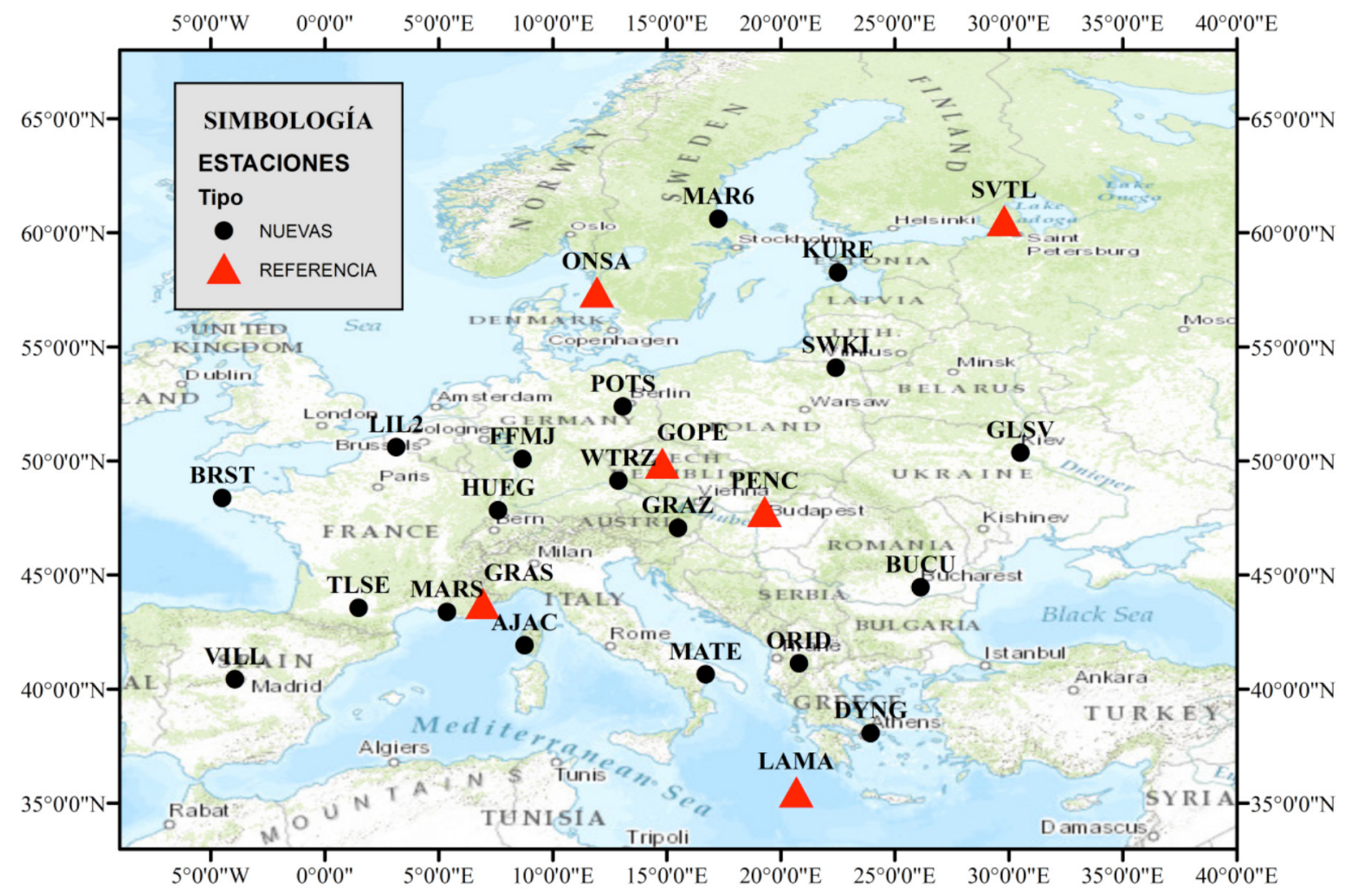

Figura 1. Diseño de la red. Elaboración propia, tomando como base el mapa "topographic" disponible en la versión 10.0 del programa ArcGis ${ }^{\circledR}$.

Artículo protegido por licencia Creative Commons: BY-NC-ND / Protected by Creative Commons: BY-NC-ND

Uniciencia es una revista de acceso abierto/ Uniciencia is an Open Access Journal. 
UNICIENCIA Vol. 32, No. 1, pp. 1-17. Enero-Junio, 2018.

\section{Descarga de datos}

Se procedió a la descarga de los datos de observación en formato RINEX. Cada estación genera un archivo de 24 horas; corresponde uno para cada día de la semana.

Para realizar el procesamiento en el software Bernese se requirió de información que indicara la trayectoria verdadera que siguieron los satélites. Para este fin se utilizaron las efemérides precisas calculadas y publicadas por el IGS tanto para GPS como para GLONASS. El uso de los EOP es necesario, pues las órbitas se deben calcular en el Sistema Inercial Celeste.

Se realizaron diferentes scripts para descargar las efemérides precisas de los sistemas GPS (junto con los EOP) y GLONASS, la solución semanal final de EUREF y con respecto al modelado ionosférico, se descargaron grillas globales que son obtenidas de CODE, ya que son requeridas para la resolución de ambigüedades por medio de la estrategia QIF.

Con respecto al modelado a priori de la troposfera, se utilizó la aplicación vmf2grd.bat, escrita por el Dr. Jorge Moya Zamora, investigador del CNPDG, para la descarga de los datos con los coeficientes de las mallas globales, las cuales son aplicadas con la Función de Mapeo de Viena (VMF).

\section{Preparación de la información en el formato de Bernese}

Para el procesamiento de los datos en el software Bernese es necesario que estén en el formato requerido por el programa. Considerando que se hizo con mediciones únicamente GPS y luego GNSS, se requirió tener dos juegos de observaciones en formato RINEX, uno con solo el registro del sistema GPS y otro con GPS y GLONASS.

Una vez descargados los datos, que están en formato Hatanaka (yyd) y convertidos a RINEX (yyO), se hizo una copia de estos a efectos de eliminar de uno de los grupos las observaciones de GLONASS y dejar únicamente las GPS, usando una rutina escrita en el lenguaje PERL por el Dr. Mauricio Gende, de la Universidad de La Plata, la cual fue adecuada para los fines del proyecto. Este proceso permite ordenar la estructura de los archivos lo que repercute en la disminución de su tamaño. Para poder ejecutarla se requiere que en la carpeta donde está el script, esté la aplicación TEQC. En la figura 2 se muestra un diagrama de este proceso.

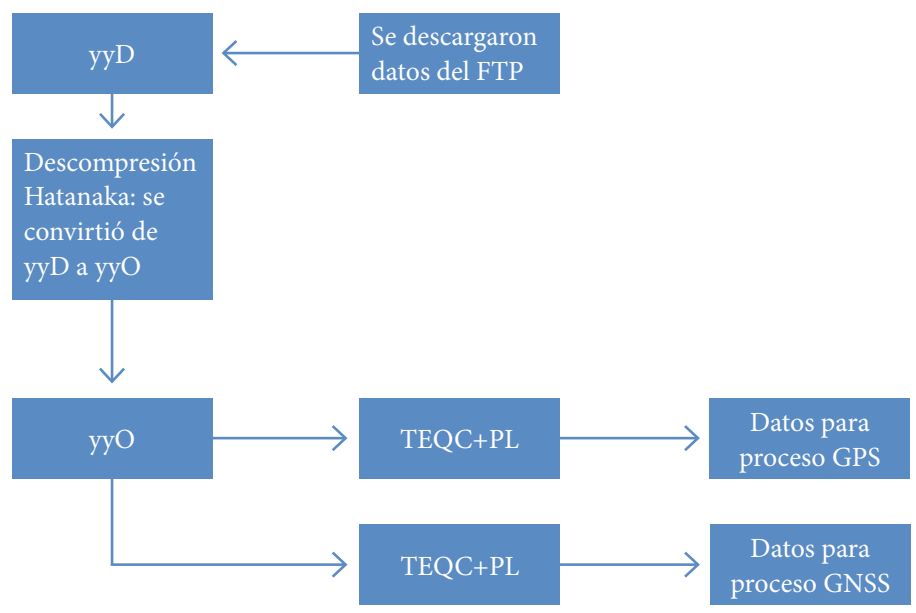

Figura 2. Proceso de la preparación de los datos. Elaboración propia. 
ISSN Electrónico: 2215-3470

DOI: http://dx.doi.org/10.15359/ru.32-1.1
UNICIENCIA Vol. 32, No. 1, pp. 1-17. Enero-Junio, 2018. URL: www.revistas.una.ac.cr/uniciencia Email: revistauniciencia@una.cr

Finalmente, es necesario generar un conjunto de archivos requeridos para el procesamiento, los cuales son:

1. Archivo de abreviaturas, requerido por Bernese para identificar las líneas bases (extensión.ABB)

2. Un archivo con la información del tipo de receptor y de antena de cada estación, así como los cambios en el equipo (extensión .STA)

3. Un archivo con coordenadas aproximadas de las estaciones (extensión .CRD)

4. Un archivo con las velocidades de las estaciones, requerido para la actualización de las coordenadas aproximadas a la época de medición (extensión.VEL)

5. El archivo con los coeficientes para modelar el efecto de la carga oceánica, en formato y con extensión .BLQ

6. El archivo con los coeficientes para modelar el efecto de la carga atmosférica (extensión.ATL).

\section{Procesamiento de los datos}

El periodo de procesamiento cubre 26 semanas del año 2014, iniciando en la semana GPS 1774 hasta la 1798; en detalle, desde el día 005, el cual corresponde al 5 de enero del 2014, hasta el 186 (5 de julio del 2014). Para el procesamiento se requiere el archivo con las correcciones a los centros de fase de las antenas. En el caso de Bernese, este archivo se denomina PHAS. Se utilizó el archivo PHAS que CODE regularmente usa. El sitio ftp para descargar el archivo PHAS es $\underline{\mathrm{ftp}: / / \mathrm{ftp} . u n i b e . c h / a i u b / B S W U S E R 52 / \mathrm{GEN} /}$

Con respecto al procesamiento de los datos se efectuó con la licencia del programa Bernese propiedad del CNPDG en su versión 5.2. Para el procesamiento de los datos se utilizó la combinación de frecuencia L3 (libre de ionósfera) con dobles diferencias y se resolvieron las ambigüedades con la estrategia QIF.

\section{Evaluación de la campaña}

Al concluir con el procesamiento, se obtiene la solución semilibre (porque las efemérides precisas se usaron como datos conocidos, aunque no hay enlace al marco de referencia terrestre).

Para evaluar la solución semilibre, se busca en el archivo .OUT de la combinación semanal, las estaciones cuyos residuales sean mayores a las tolerancias establecidas (las cuales, para este proyecto son de $\pm 10 \mathrm{~mm}$ en la componente norte y este y $\pm 20 \mathrm{~mm}$ en la componente up), conocidos como outliers. Si ocurre que alguna estación presenta un residual mayor a la tolerancia, es necesario eliminar el archivo RINEX de ese día para la misma y volver a procesar el día. También se revisa que las correcciones a las coordenadas aproximadas no sean mayores a $5 \mathrm{~cm}$, en caso de que lo sean, se corrige el archivo de coordenadas para el procesamiento de la semana siguiente y en caso de que sean mayores a $10 \mathrm{~cm}$ se corrige el archivo y se vuelve a procesar la semana en cuestión.

\section{Definición de las estaciones de referencia}

Como se comentó en el punto anterior, producto del procesamiento se obtiene una solución semilibre. Para efectos de análisis, es necesario enlazar la red a un marco de referencia, por lo que, para efectuar este proceso, se requiere definir estaciones de referencia, conocidas como 
estaciones fiduciales. Para la definición de las estaciones fiduciales se realizó un primer análisis, el cual consistió en determinar, dentro de la solución de EUREF, cuáles son utilizadas como referencia en la combinación efectuada para la EPN. En la nomenclatura que utiliza el programa Bernese se puede asignar un "flag" de forma que las clasifica basado en criterios definidos por el usuario. El flag W representa estaciones cuyas coordenadas fueron estimadas con alguna restricción en la combinación de las ecuaciones normales mientras que el flag A son estaciones cuya posición fue estimada sin restricción alguna.

Con base en lo anterior se decidió usar como estaciones fiduciales para el proyecto estaciones con flag W. El segundo criterio se basó en la distribución espacial de las estaciones. Como resultado de la aplicación de estos criterios se seleccionaron seis estaciones: GOPE, GRAS, LAMA, ONSA, PENC y SVTL.

\section{Generación de la solución final}

Una vez aceptada la campaña se procede a introducir el marco de referencia para obtener la solución final semanal del proyecto. Para la presente investigación, se usaron para las estaciones fiduciales las coordenadas producto de las soluciones semanales finales generadas por EUREF. Esta información se traslada a un archivo con coordenadas para la red del proyecto, el cual es usado al momento de generar la solución semanal final con el programa Bernese 5.2.

\section{Evaluación de la solución final}

Con el fin de evaluar y aceptar los resultados finales del procesamiento de la red usada en el proyecto se efectúan tres comparaciones basadas en la transformación de Helmert:

1. Se compara las coordenadas de referencia con la solución final semanal del proyecto, con el fin de determinar la consistencia entre las coordenadas de las estaciones fiduciales. En esta comparación solo se usan las estaciones usadas como referencia. Al archivo resultante se le denomina RCSFWWWW, donde WWWW es la semana GPS; la tolerancia para aceptar los resultados es de $\pm 4 \mathrm{~mm}$, esto de acuerdo con la consistencia de la solución multianual EUREF con respecto al IGS08.

2. Se compara la solución semilibre semanal con la solución final semanal; esta se realiza con el fin de determinar las deformaciones en la red al introducir el marco de referencia (generar la solución final semanal del proyecto). El archivo resultante fue nombrado como SLSFWWWW; la tolerancia para aceptar los resultados es de $\pm 8 \mathrm{~mm}$ horizontal $\mathrm{y} \pm 16 \mathrm{~mm}$ vertical, esto basado en los criterios aplicados por el centro de combinación de EUREF para evaluar su solución.

3. Se compara la solución de EUREF con la solución final del proyecto; esto se realiza con el fin de determinar la consistencia externa de los resultados (la consistencia de la solución de la red del proyecto comparada con el marco de referencia europeo); el archivo de salida fue nombrado como SFEUWWWW, donde WWWW es la semana GPS; la tolerancia para aceptar los resultados es de $\pm 2 \mathrm{~mm}$ horizontal y $\pm 5 \mathrm{~mm}$ vertical; esto basado en la calidad de la solución multianual de EUREF.

La metodología descrita anteriormente para evaluar la solución final es la implementada en el Centro Nacional de Procesamiento de Datos GNSS de la ETCG-UNA. 
ISSN Electrónico: 2215-3470

DOI: http://dx.doi.org/10.15359/ru.32-1.1
UNICIENCIA Vol. 32, No. 1, pp. 1-17. Enero-Junio, 2018. URL: www.revistas.una.ac.cr/uniciencia Email: revistauniciencia@una.cr

\section{Resultados}

\section{Comparación de los resultados entre el procesamiento GPS con el procesamiento GNSS}

A continuación, se muestran los resultados de la comparación entre los procesamientos GPS y GNSS. Entre los parámetros que se analizaron está el RMS de la repetibilidad de las coordenadas, los valores absolutos de las coordenadas y la estimación del retardo troposférico zenital.

\section{Repetibilidad}

Para evaluar la calidad de los resultados del procesamiento hay dos opciones: la primera, usar la información de la matriz varianza y covarianza de las coordenadas; la segunda, analizar los valores de la repetibilidad diaria. A pesar de que la desviación estándar es el primer indicador de la calidad del procesamiento, por lo general proporciona valores muy optimistas por la calidad de los parámetros; sin embargo, la repetibilidad proporciona una medida más realista de la precisión de las coordenadas de la estación y es un indicador de la dispersión de las soluciones de todos los días (Nocquet, 2008).

Para analizar los valores de la repetibilidad obtenidos y poder realizar el análisis, se elaboró una rutina en el programa MATLAB. Para utilizar la rutina se tabularon los valores que se extraen de los resúmenes de las soluciones semanales (archivos extensión. SUM). La figura 3 muestra las repetibilidades de las soluciones finales del procesamiento GPS y GNSS realizados en el proyecto y la figura 4 muestra la comparación de las repetibilidades de la solución final del procesamiento GNSS y la generada por EUREF.
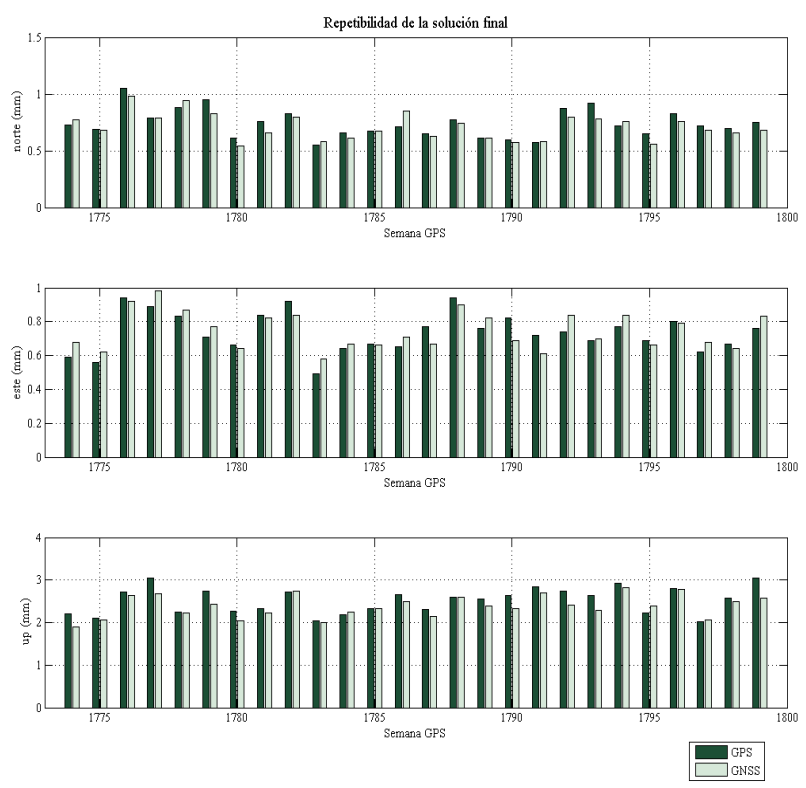

Figura 3. Repetibilidad de la solución final GPS y GNSS. Fuente propia del estudio. 
UNICIENCIA Vol. 32, No. 1, pp. 1-17. Enero-Junio, 2018.
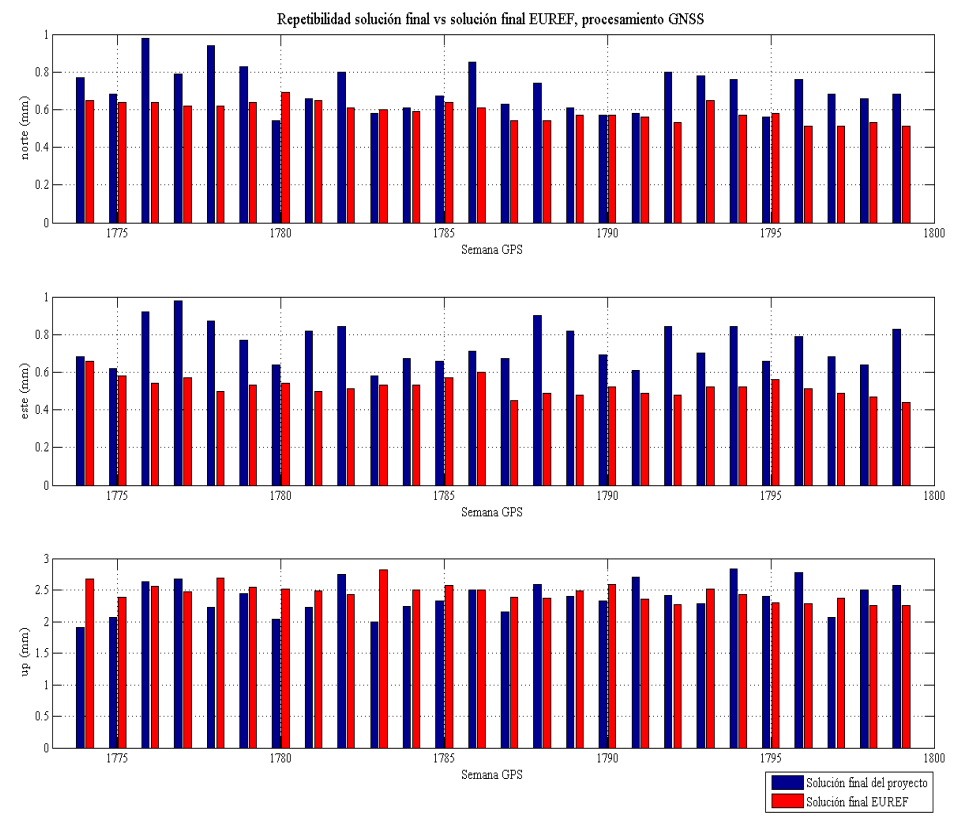

Figura 4. Repetibilidad de la solución final del proyecto vs solución final EUREF. Fuente propia del estudio.

\section{Coordenadas}

Para comparar las soluciones finales del procesamiento GPS y GNSS, se utilizó una rutina en Matlab llamada series.m, la cual genera un gráfico para las componentes norte, este y up de cada estación, esta requiere del archivo de texto "datos.txt", el cual contiene las coordenadas en el sistema topocéntrico de GPS y GNSS para el sitio que se desea comparar. En las figuras 5 y 6 se muestran los resultados de esta comparación para la estación AJAC y SVTL, respectivamente.
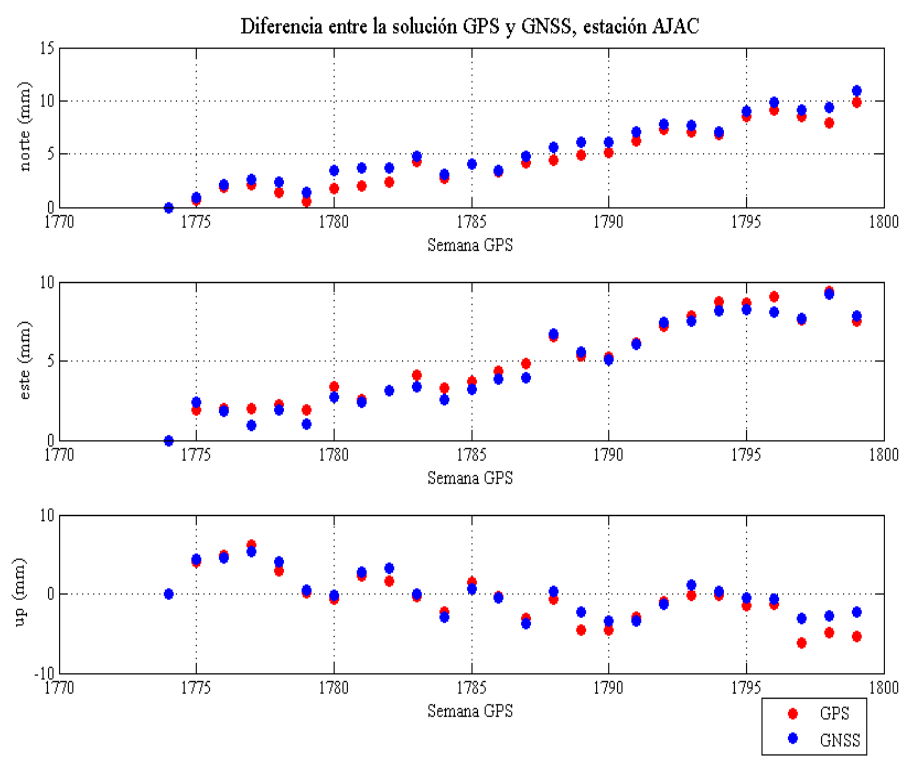

Figura 5. Diferencia entre la solución GPS y GNSS para la estación AJAC. Fuente propia del estudio. 

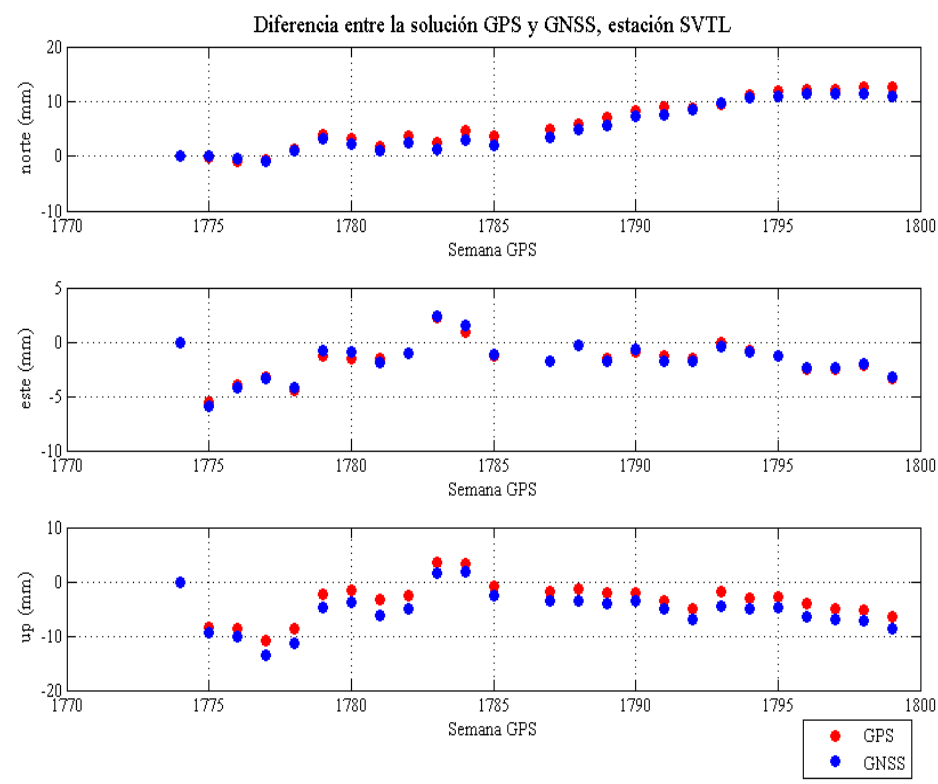

Figura 6. Diferencia entre la solución GPS y GNSS para la estación SVTL. Fuente propia del estudio.

\section{Tropósfera}

Para el análisis de la variación del ZTD, se tabuló este valor obtenido tras el procesamiento de datos GPS y GNSS en un archivo de Excel para las estaciones LAMA, MAR6 y WTZR. Estas fueron seleccionadas debido a su ubicación en la red; MAR6 es la que se encuentra más al norte, WTZR, es la que se está más al centro y LAMA es la que se localiza más cerca de la línea del Ecuador. En la figura 7 se puede observar el valor del ZTD para ambos procesamientos de la estación LAMA.

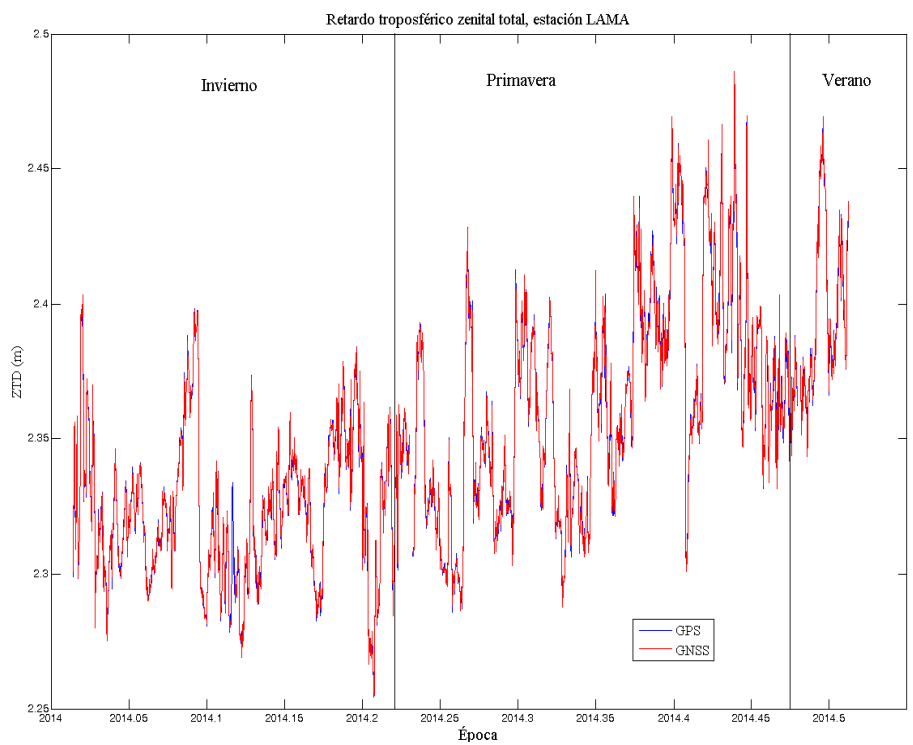

Figura 7. ZTD estación LAMA. Fuente propia del estudio. 
UNICIENCIA Vol. 32, No. 1, pp. 1-17. Enero-Junio, 2018.

Email: revistauniciencia@una.cr

En las figuras 8 y 9 se muestran la comparación de parámetros vinculados al procesamiento de los datos.

Número de observaciones en los procesamientos efectuados

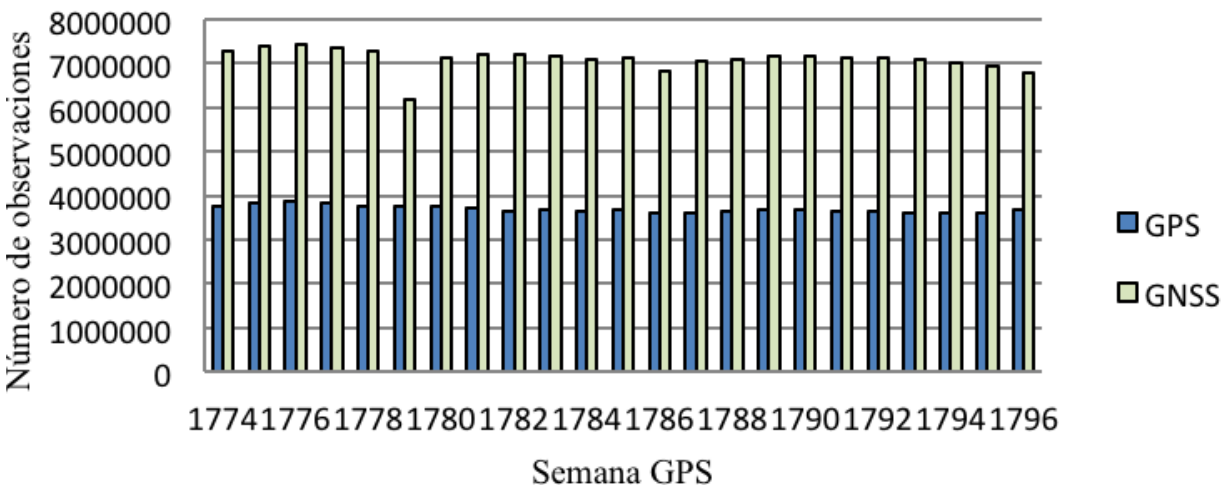

Figura 8. Número de observaciones en los procesamientos efectuados. Fuente propia del estudio.

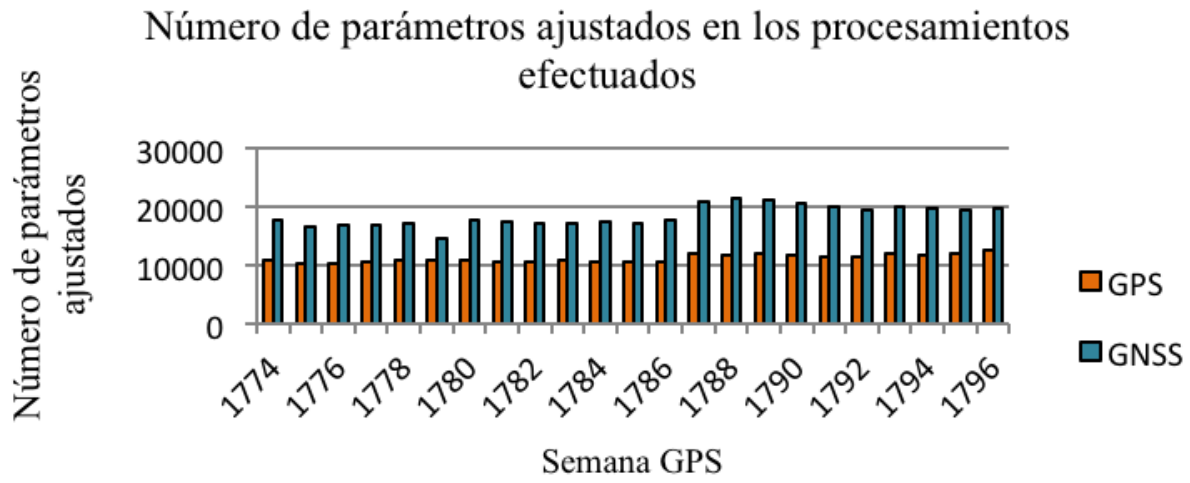

Figura 9. Número de parámetros ajustados en los procesamientos efectuados.

\section{Análisis de resultados}

En la figura 3 se observa que la repetibilidad de la solución final GPS y de la solución final GNSS es similar en las tres componentes (norte, este y up); la máxima diferencia entre las soluciones finales es de 0,5 $\mathrm{mm}$ en la componente up en la semana 1799.

Con respecto a las coordenadas en la figura 5 se puede observar la comparación de la solución final del procesamiento GPS y GNSS para la estación AJAC en la cual se puede apreciar que la diferencia máxima es:

1. Para la componente norte, $-1,72 \mathrm{~mm}$ en la semana 1781 .

2. En el este es de $1,06 \mathrm{~mm}$ en la semana 1777 .

3. En up es de $-3,08 \mathrm{~mm}$ en la semana 1797.

De igual manera en la figura 6 se muestra la comparación entre las soluciones finales del procesamiento GPS y GNSS para la estación de referencia SVTL, se puede apreciar que las diferencias tienden a cero, la mayor que se destaca es: 
1. En el componente norte es en la semana 1785 con $1,69 \mathrm{~mm}$.

2. En el este es en la semana 1784 con $-0,62 \mathrm{~mm}$.

3. En up es en la semana 1781 con $2,92 \mathrm{~mm}$.

Se puede observar en la figura 7 que el ZTD de la estación LAMA tiene un comportamiento oscilante, ya que este va desde los 2,25 m hasta los 2,48 m, además se obtiene el mismo resultado en el procesamiento GPS y en el GNSS. A pesar de la variación se puede apreciar que existe una tendencia con respecto al comportamiento del ZTD, ya que este progresivamente va incrementando su valor; esto es esperado, debido a que se encuentra en una latitud cercana al Ecuador.

Se observa, al comparar las repetibilidades de las soluciones del procesamiento GNSS y las de EUREF, que hay una alta consistencia entre ellas; esto se corrobora cuando se calculan las diferencias entre los valores de ambas soluciones, las cuales tienen un promedio de $0,12 \mathrm{~mm}$ en el componente norte, $0,23 \mathrm{~mm}$ en el componente este y 0,08 $\mathrm{mm}$ en el componente vertical.

En otros análisis, el número de observaciones para el procesamiento GNSS es en promedio 92\% más que la cantidad de observaciones del procesamiento GPS. Esto se debe a que en el procesamiento GNSS se incluyen las observaciones del sistema GLONASS. De igual manera el procesamiento de datos GNSS tiene en promedio 64\% más parámetros en comparación con el procesamiento de datos GPS, debido a que incluye las ambigüedades GLONASS.

El tamaño de los archivos con los datos GNSS son prácticamente el doble en relación con el tamaño que los archivos con datos únicamente GPS, debido a que estos archivos de observación poseen los registros de los sistemas GPS y GLONASS, mientras que los archivos GPS solo poseen observaciones del sistema GPS.

También se evidenció la diferencia en el tiempo de procesamiento entre las campañas GPS y las GNSS, ya que el procesamiento GPS tienen un tiempo de procesamiento de entre $1 \mathrm{~h} 17 \mathrm{~m}$ y $1 \mathrm{~h} 25 \mathrm{~m}$ mientras que el procesamiento GNSS tiene un tiempo de procesamiento promedio de $2 \mathrm{~h} 58 \mathrm{~m}$.

\section{Conclusiones}

A pesar de la inclusión de las observables GLONASS en un procesamiento combinado con GPS, las cuales repercuten directamente en la cantidad total de observaciones e incógnitas a estimar, y luego de la comparación de las coordenadas obtenidas para la red en ambos cálculos, así como el estudio del valor del ZTD estimado para tres estaciones y otros parámetros, se concluye que los resultados de un procesamiento GNSS son equivalentes al de GPS. Esto quiere decir que, en la actualidad, la inclusión de las señales GLONASS no mejora significativamente ni desmejora la calidad de los resultados del procesamiento con únicamente datos GPS.

Con la creación del proyecto SIRGAS-GLONASS se demostró, en el año 2013, que los resultados del procesamiento GNSS utilizando Bernese versión 5.0 tienen la tendencia a ser menos precisos que los resultados del procesamiento de únicamente datos GPS. Tras el procesamiento GPS y GNSS y los resultados obtenidos en el presente proyecto de graduación, utilizando el programa Bernese 5.2, se observó que el programa Bernese mejoró el tratamiento de la señal GLONASS, ya que los resultados de ambos procesamientos son equivalentes entre sí.

Las soluciones semilibres tanto para el procesamiento GPS como el GNSS, son similares entre sí, lo cual significa que la geometría es consistente en los dos procesamientos, ya que el valor de la repetibilidad promedio de ambos procesamientos difiere en la componente norte $0,02 \mathrm{~mm}$, en la componente este $0 \mathrm{~mm}$ y en la componente up $0,16 \mathrm{~mm}$. 
UNICIENCIA Vol. 32, No. 1, pp. 1-17. Enero-Junio, 2018.

URL: www.revistas.una.ac.cr/uniciencia

Email: revistauniciencia@una.cr

Producto del procesamiento semanal se obtiene, en primera instancia, la solución semilibre que tiene la característica de que las coordenadas obtenidas tras el procesamiento son coordenadas libres en el espacio, por lo que es requerido introducir el marco de referencia para asociarlas con el sistema de coordenadas terrestre; lo anterior tiene como consecuencia que la red se deforma. Se busca, entonces, que esta deformación sea mínima.

Al introducir el marco de referencia se concluye que la red no sufre una deformación significativa debido a que el promedio del RMS de los residuos en ambos procesamientos es en el componente norte $0,99 \mathrm{~mm}$, en el componente este es $0,88 \mathrm{~mm}$ y en el componente up es 0,94 $\mathrm{mm}$.

Para efectuar el análisis de los resultados del procesamiento se escribieron rutinas en el programa $\mathrm{Matlab}^{\oplus}$, ya que este programa es una herramienta útil para las ciencias porque facilita la programación al tener un lenguaje sencillo. Las rutinas escritas permitieron generar gráficos con información sobre el porcentaje de ambigüedades resueltas, la repetibilidad de las soluciones, generación de series temporales y el análisis del ZTD.

Para evaluar la confiabilidad de las soluciones finales y para determinar las variaciones de las coordenadas de referencia con respecto a la solución final, se efectúa una transformación de Helmert, donde se comparan las coordenadas de referencia con la solución final; para determinar las deformaciones en la red al introducir el marco de referencia se compara la solución semilibre con la solución final y para determinar la confiabilidad externa de la red se compara la solución final de EUREF con la solución final del proyecto. Estas comparaciones permiten concluir sobre la alta calidad del procesamiento efectuado y tener confiabilidad en las coordenadas obtenidas.

Luego de la comparación con las soluciones finales de EUREF, se observa que la solución de EUREF tiene una calidad levemente mejor en los componentes norte y este con respecto a la red del proyecto; sin embargo, entre ambas soluciones hay una alta consistencia en el componente vertical. No se debe olvidar que cada estación de EUREF es procesada por al menos tres centros de análisis, los que repercute en una redundancia en las soluciones.

\section{Referencias}

Agria, J., Altamini, Z., Boucher, C., Brockmann, E.. Caporali, A,. Gurtner, W., Habrich, H., Hornik, H., Ihde, J., Kenyeres, A., Mäkinen, J., Marel, H., Seeger, H., Simek, Jaroslav., Stangl, G. y Weber, G. (2009). Status of the European Reference Frame(EUREF). International Association of Geodesy Symposia, 133, 47-56. doi https://doi.org/10.1007/978-3-540-85426-5 6

Altamimi, Z., Collilieux, X. y Métivier, L. (2011). ITRF2008: an improved solution of the international terrestrial reference frame. Journal of Geodesy, 85(8), 457-473. doi: https://doi. org/10.1007/s00190-011-0444-4

Angermann, D., Drewes, H., Krügel, M., Meisel, B., Gerstl, M., Kelm, R., Müller, H., Seemüller, W. y Tesmer, V. (2004). ITRS Combination Center at DGFI: A Terrestrial Reference Frame Realization. Recuperado de http://www.dgk.badw.de/fileadmin/docs/b-313.pdf

Bruyninx, C. (2006). Comparing GPS-only with GPS + GLONASS positioning in a regional permanent GNSS network. GPS Solutions, 11(2), 97-106. doi https://doi.org/10.1007/ s10291-006-0041-9

Dach, R. y Walser, P. (2013). Bernese GNSS Software Version 5.2. Course Tutorial. Berna: Stampfli Publications AG. Recuperado de http://www.bernese.unibe.ch/docs/TUTORIAL.pdf 
Dach, R., Hugentobler, U., Fridez, P. y Meindl, M. (2007). Bernese GPS Software Version 5.0. Berna: Stampfli Publications AG.

Habrich, H. (2009). Evaluation of analysis options for GLONASS observations in regional GNSS networks. Geodetic Reference Frames, International Association of Geodesy Symposia, 134, 121-129. Berlin, Alemania: Springer-Verlag Berlin Heidelberg. doi https://doi. org/10.1007/978-3-642-00860-3 19

Hofmann - Wellenhof, B., Lichtenegger, H. y Wasle, E. (2008). GNSS - Global Navigation Satellite Systems. Austria: Springer-Verlag Wien.

Nocquet, J., Mothes, P. y Alvarado, A. (2008). Geodesia, geodinámica y ciclo sísmico en Ecuador. Geología y geofísica marina y terrestre del Ecuador desde la costa continental hasta las islas Galápagos, 1, 83-94. Recuperado de http://renag.unice.fr/regal/PERSO/JMN/publis/ geodesia ecuador texto y figuras.pdf

Petit, G. y Luzum, B. (2010). IERS Conventions. Alemania: Verlag des Bundesamtes für kartographie und Geodäsie.

Rebischung, P., Griffiths, J., Ray, J., Shmid, R., Collilieux, X. y Garayt, B. (2011). IGS08: the IGS realization of ITRF2008. GPS Solutions, 16(4), 485-493. doi: https://doi.org/10.1007/ s10291-011-0248-2

Schmid, R., Steigenberger, P., Gendt, G., Ge, M. y Rothacher, M. (2007). Generation of a consistent absolute phase center correction model for GPS receiver and satellite antennas. $J$ Geod Geoinf, 81(12), 781-798. doi: https://doi.org/10.1007/s00190-007-0148-y

Seeber, G. (2003). Satellite Geodesy. Berlin, Alemania: Walter de Gruyter. Doi https://doi. org/10.1515/9783110200089

Torge, W. (2001). Geodesy. Berlin, Alemania: Walter de Gruyter. Doi https://doi. org $/ 10.1515 / 9783110879957$

Walser, P. (2014). Bernese GNSS Software. Bernese GNSS Software. Recuperado de http://www. bernese.unibe.ch/

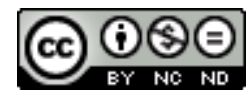

Análisis de la influencia de las señales del sistema GLONASS en el procesamiento de una red geodésica regional (Diana Paniagua-Jiménez y otros) por Revista Uniciencia se encuentra bajo una Licencia CreativeCommons AtribuciónNoComercial-SinDerivadas 3.0 Unported. 